\title{
Transcriptional memory remodelled
}

DOI:

10.1038/nrg2110

URLs

Entrez

GAL1

http://www.ncbi.nlm.nih.gov/ entrez/query.fcgi?db=gene\&c $\mathrm{md}=$ Retrieve\&dopt=full report\&list_uids $=852308$

SWI2

http://www.ncbi.nlm.nih.gov/ entrez/query.fcgi?db=gene\&c $\mathrm{md}=$ Retrievegdopt=full reportElist_uids $=854465$

Craig Peterson's web site: http://www.umassmed.edu/ $\mathrm{pmm} /$ faculty/peterson.cfm
For cells to respond effectively to their environment, forming a 'memory' of transcriptional responses to certain conditions can be a useful strategy. It is generally believed that histone modifications have a role in this type of mechanism. Now, work in yeast reveals that ATP-dependent chromatin remodelling enzymes provide a second form of epigenetic transcriptional memory.

The Saccharomyces cerevisiae GAL1 gene product allows cells to use galactose as an energy source. Kundu and colleagues found that when cells are first exposed to galactose, GAL1 expression takes more than an hour to reach its peak. However, if cells are grown in the presence of galactose, followed by repression of GAL1 expression by glucose and then reexposure to galactose, transcription

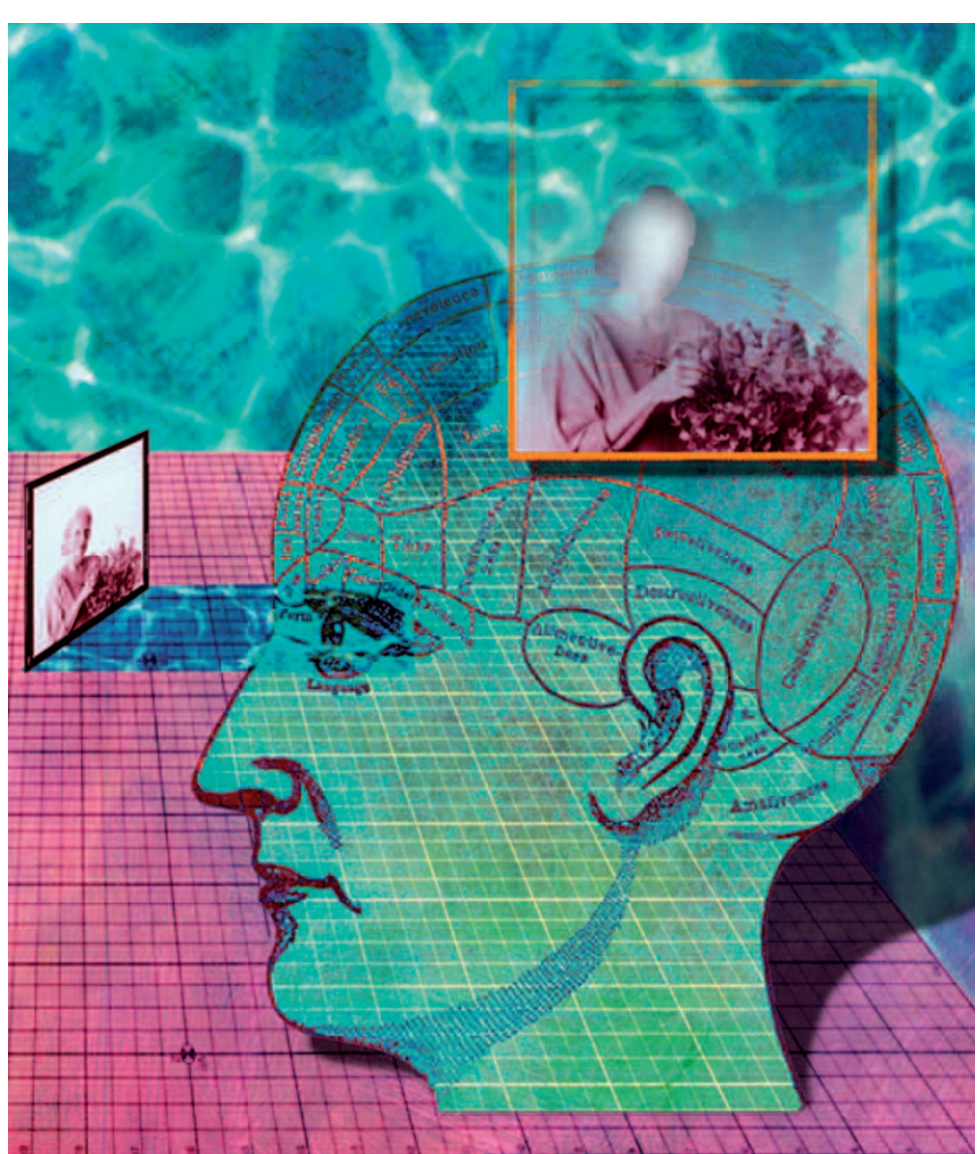

at the GAL1 locus resumes rapidly, peaking in less than 10 minutes. The authors propose that yeast cells remember their initial encounter with galactose and can somehow reactivate GAL1 expression more quickly. This response is maintained through at least one cell division, suggesting that a form of epigenetically inherited transcriptional memory is at work.

By tracking protein recruitment at GAL1, the authors ruled out the possibility that components of the transcriptional machinery are responsible for this epigenetic memory. They also eliminated roles for several histone-modifying enzymes that might be considered as likely candidates for mediating the memory: deletions of the genes encoding these proteins had no effect on the re-induction of GAL1. the SWI2 gene, which encodes the SWI/SNF ATP-dependent remodelling enzyme, was involved. Deletion of this gene has no effect on steadystate GAL1 expression, but abolishes the rapid re-induction of GAL1 after a second exposure. This effect is not simply due to a general need for SWI/SNF in antagonizing glucose repression, as GAL1 transcripts are eventually made in swi $\Delta$ mutants.

Kundu and colleagues propose that SWI/SNF is needed after initial GAL1 transcription to establish a heritable epigenetic state that allows rapid reinduction. Using a strain carrying a mutation in the ATPase domain, they showed that the enzymatic activity of SWI/SNF is essential for establishing this state.

Previous studies have shown that an ATP-dependent remodeller of the ISWI family - members of which are generally associated with transcriptional repression - is recruited to $G A L 1$ during transcriptional induction. When the swi $2 \Delta$ mutation was combined with deletions of either of the S. cerevisiae genes that encode ISWI-like enzymes, rapid GAL1 re-induction was abolished. On this basis, the authors propose a model in which SWI/SNF is needed to prevent an ISWI-mediated return of chromatin at GAL1 to a transcriptionally repressive state.

ISWI-like enzymes and SWI/SNF are already known to have antagonistic effects at several other genes. Future investigations at these and other loci should reveal whether transcriptional memory involving ATP-dependent remodelling is a widespread phenomenon in yeast and other organisms.

Louisa Flintoft

ORIGINAL RESEARCH PAPER Kundu, S., Horn, P. J. \& Peterson, C. L. SWI/SNF is required for transcriptional memory at the yeast GAL gene cluster. Genes Dev. 21, 997-1004 (2007) WEB SITE

Craig Peterson's web site: http://www. umassmed.edu/pmm/faculty/peterson.cfm 